\title{
Quantitative aspects of the Galperin $L$ parameter
}

\author{
J. C. Kosik \\ Centre de Données de la Physique des Plasmas, Av. du Colonel Roche, 31400 Toulouse, France
}

Received: 1 March 2007 - Revised: 5 September 2007 - Accepted: 30 October 2007 - Published: 2 January 2008

\begin{abstract}
A new geomagnetic parameter was suggested twenty years ago by Y. Galperin, the Galperin $L$ parameter, and it was introduced into the CNES Maglib for FrenchRussian projects in the exploration of the distant magnetosphere. The definition and the advantages of the Galperin $L$ parameter are recalled in this brief paper. Unforeseen possibilities in the use of this parameter for mathematical models of the magnetosphere are stressed using past results obtained with the Mead model. The Galperin $L$ parameter is shown to add, in the synchronous region, a quantitative capability to the qualitative description (labelling) of the magnetosphere. More work will be necessary to adapt past mathematical models to present numerical models and extend the domain of the quantitative applications of the Galperin $L$ parameter.
\end{abstract}

Keywords. Magnetospheric physics (Magnetospheric configuration and dynamics, General or miscellaneous)

\section{Introduction}

Corrected geomagnetic coordinates were introduced in the 1950s to better organize data in the high-latitude regions. Hultquist (1958a) calculated the spherical harmonic coefficients of the internal magnetic field of the Earth in a centered dipole coordinate system. In a later work, Hultquist (1958b) calculated the deviations of the real field line from the dipole field line due to the perturbation of the higher spherical harmonic coefficients. The integrated deviations along the dipole field line gave the displacement vectors in the Northern and Southern hemispheres. The corrected geomagnetic coordinates of a point were the dipole geomagnetic coordinates corrected by the displacement vector. Hakura (1965) improved the method by tracing the field line down to the geomagnetic equator using an IGRF magnetic field. From this location a dipole field line was traced back to the Earth, giving the corrected geomagnetic location of the point. More recently, Stasiewicz (1991) extended Hakura's solution by combining internal and external fields. From a point in space the tracing down to the Earth is performed using the total magnetic field (IGRF + the Kosik 1998 or Tsyganenko 1989 models), then from this conjugate point on Earth, the field line tracing down to the geomagnetic dipole equator uses the internal magnetic field only. These efforts presumably led Y. Galperin to introduce for the high altitude and high inclination magnetospheric spacecraft a modern shell parameter in replacement of the McIlwain $L$ parameter. This parameter was introduced in the CNES Maglib. The Maglib software includes routines for the calculation of the internal magnetic fields, external magnetic fields, transformation matrices between different frames of reference and the calculation of geophysical parameters.

The Maglib was developed for the treatment of data from the Interball and Cluster spacecraft (Hapgood et al., 2004). At the same time, Prokhorenko (1995) introduced this parameter at IKI (Cosmic Research Institute, Moscow), for the French-Russian planning of the Interball experiments. In this short paper we recall the definition of the Galperin $L$ parameter and its ability to label fluxes in the outer magnetosphere. We show that this parameter has a major advantage over the choice of an equatorial labelling, as we demonstrate its direct link with past results obtained with a simplified mathematical model of the magnetosphere in the synchronous region. The Galperin $L$ parameter also has a quantitative capability in this region.

Correspondence to: J. C. Kosik

(jean-claude.kosik@wanadoo.fr)

Published by Copernicus Publications on behalf of the European Geosciences Union. 


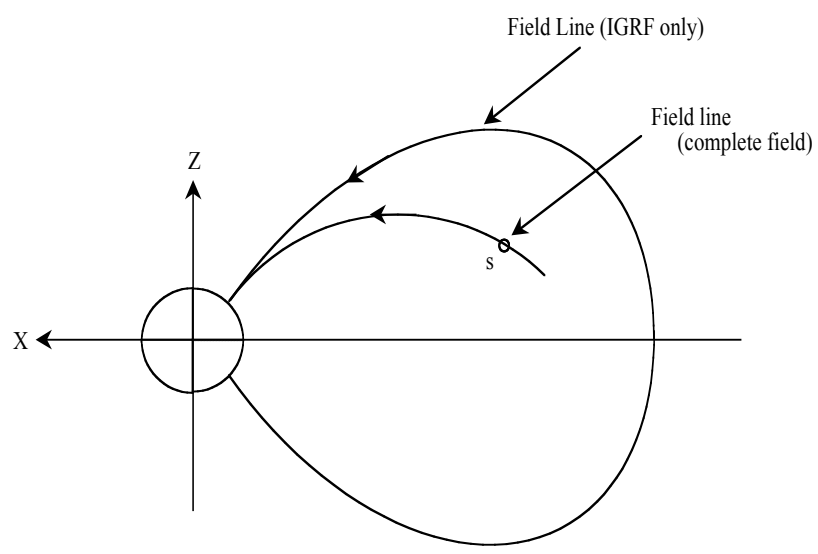

Fig. 1. From the spacecraft location $s$ the field line is traced down to the Earth using the total field (IGRF + external magnetic field). From the conjugate point a field line is traced using the IGRF magnetic field only and the McIlwain $L$ parameter is calculated along this field line for this conjugate point.

\section{The McIlwain $L$ parameter}

Northrop and Teller (1960) have shown that the motion of charged particles could be described using adiabatic invariants. In the absence of perturbations the motion of the particles can be described by the first two invariants, $\mu=E / B_{m}$ and $J=2 p I$, where $E$ is the the energy of the particle, $p$ the momentum and $B_{m}$ the magnetic field intensity at the mirror point. $I$ is the path integral between the mirror points $M, M^{\prime}$ :

$I=\oint_{M M^{\prime}} \sqrt{1-\frac{B}{B_{m}}} d s$.

The points in space that have the same value of $B_{m}$ and $I$ form a ring in each hemisphere and a particle bouncing beween the mirror points will drift along a shell described by the field lines that connect these rings. In a dipole field particles that have different mirror points on the same field line will drift in longitude to the same field line. In the Earth's heterogeneous magnetic field this will not be the case and two particles that have two different mirror points along the same field line of force will drift in longitude to different lines of force. But this effect is relatively small, as was discussed in detail by Roederer (1970). Taking into account the properties of the dipole magnetic field, Mcllwain (1961) introduced a smoothing parameter $L$, defined for a point in the Earth's magnetic field by the equation:

$L^{3} B_{m} / M=F\left(I^{3} B_{m} / M\right)$,

where $M$ is the dipole moment of the Earth. Mcllwain presented the function $F$ in a table of 125 values and as a set of polynomials of the form

$\operatorname{Ln}\left(L^{3} B_{m} / M-1\right)=\sum_{0}^{6} c_{n}\left(\operatorname{Ln} I^{3} B_{m}, / M\right)^{n}$

where $I$ and $B_{m}$ are to be calculated with a representation of the Earth's field. The $B L$ coordinate system is perfectly adequate for the inner magnetosphere (equatorial distance less than $4 \mathrm{Re})$.

\section{The Galperin $L$ parameter}

For the outer magnetosphere, shell splitting from external magnetic sources is more and more important with the increase in the distance to the Earth. The shell splitting due to the multipoles of the magnetic field of the Earth can be neglected with respect to this outer zone shell splitting. The Mcllwain parameter is no longer an adequate parameter. Roederer (1970) suggested other labelling techniques, using, for example, the third adiabatic invariant. Galperin suggested the following recipe for a new $L$ parameter.

From the point $s$ of the measurement

- trace the field line down to the Earth with the complete internal plus external magnetic field

- for the conjugate point calculate the usual McIlwain $L$ parameter using the internal field only.

This technique is illustrated in Fig. 1. It can be applied even in the case of open field lines as the first step is the tracing down to the Earth.

In some sense, the Galperin $L$ parameter can be considered as a geomagnetically corrected McIlwain $L$ parameter.

\section{The Galperin $L$ parameter and the labelling of direc- tional fluxes in the outer zone}

If we consider a bunch of particles of very small pitchangle measured in the equatorial plane along a distorted field line, this bunch of particles will follow the field line until it reaches the mirror point and then bounces back. The directional fluxes associated with these particles can be measured along the field line. If one uses the usual McIlwain $L$ parameter which can be approximated in the outer zone to the dipole $L$, the labelling of these directional fluxes can be obtained by tracing dipole field lines through the measurement locations. To each location a different dipole field line is associated, thus to the measurements of this bunch of particles are associated a series of dipole $L$ values, and more precisely, a continuum of $L$ values between $L_{\min }$ and $L_{\max }$. $L_{\min }$ corresponds to the measurement at the equator and $L_{\max }$ corresponds to the measurement at the mirror point. 
If we now use the Galperin $L$ method, we trace the real field line down to the Earth, i.e. we follow the bunch of particles along its bounce, then we trace a dipole field line (approximation of the internal field in the outer zone) from the Earth down to the equator and obtain the Galperin $L$ parameter nearly equal to $L_{\text {max }}$. In this case all the measurements of this bunch of particles have the same label, L. In Fig. 2 , the bunch of particles is represented as an arrow. The McIlwain parameter for each measurement along the real field line is obtained in this outer region of the magnetosphere by tracing a dipole field line through the point of measurement. We obtain a series of quasi-McIlwain $L$ values between 5.2 and 7. The Galperin $L$ parameter corresponds to the outermost dipole field line and has a value of 7 . This value will be the same for all the measurements along the real field line.

\section{The Galperin $L$ parameter and its quantitative as- pects}

During the past 50 years, several mathematical models of the magnetosphere have been built, from the Mead model to the recent models Kosik (1998) or Tsyganenko (1989). For the sake of simplicity and for the ease of demonstration we will refer specifically to the results obtained using the Mead model.

Ignoring the higher order harmonics, Mead (1964) showed that the outer magnetosphere can be described with three potential terms :

$V=\frac{1}{r^{2}} g_{1}^{0} \cos \theta+r \bar{g}_{1}^{0} \cos \theta+\frac{\sqrt{3}}{2} r^{2} \bar{g}_{2}^{1} \sin 2 \theta \cos \varphi$.

In this expression $r, \theta, \varphi$ are the spherical coordinates, $r$ is expressed in Earth radii, $\theta$ is the colatitude measured from the north magnetic axis, and $\varphi$ the longitude counted from the noon meridian. The first term is the dipole magnetic field ( $g_{1}^{0}=-0.31$ Gauss), the second term is an axisymmetric compression term $\left(\bar{g}_{1}^{0}=-0.00025\right.$ gauss $)$ and the third term is the noon-midnight asymmetry term $\left(\bar{g}_{2}^{1}=-0.000012\right.$ Gauss). The magnetic field components can be derived by taking the gradient $\vec{B}=-\overrightarrow{\nabla V}$

$$
\begin{aligned}
& B_{r}=\frac{2}{r^{3}} g_{1}^{0} \cos \theta-g_{1}^{0} \cos \theta-\sqrt{3} r \bar{g}_{2}^{1} \sin 2 \theta \cos \phi \\
& B_{\theta}=\frac{1}{r^{3}} g_{1}^{0} \sin \theta+\bar{g}_{1}^{0} \sin \theta-\sqrt{3} r \bar{g}_{2}^{1} \cos 2 \theta \cos \varphi \\
& B_{\varphi}=\sqrt{3} r \bar{g}_{2}^{1} \cos \theta \sin \varphi .
\end{aligned}
$$

Inspection of Eq. (5b) for $\theta=\pi / 2$ shows that the second term $\bar{g}_{1}^{0}$ is an axisymmetric field added to the dipole field, while the third term $\bar{g}_{2}^{1}$ compresses the dipole field at noon and depresses the dipole field at midnight.

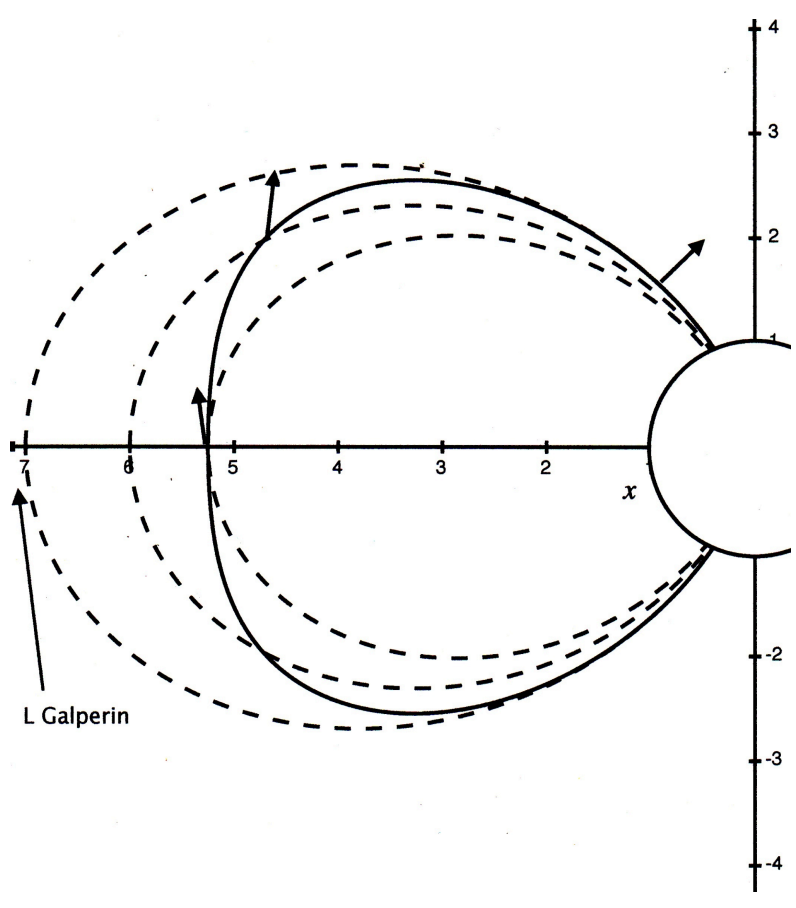

Fig. 2. Arrows represent the directional fluxes of a bunch of particles mirroring near the Earth. For the outer zone the McIlwain $L$ can be approximated by the intersections of the dipole field lines with the equator. The directional fluxes have different McIlwain $L$ parameters depending on the latitude. There is only one Galperin $L$ parameter which corresponds to the distance of the intersection of the outermost dipole field line with the equator, a distance expressed in Earth radii.

It is possible to derive the field lines equations using a linearization technique, from which we obtain (Kosik, 1971a)

$$
\begin{aligned}
r= & L \sin ^{2} \theta\left\{1-\frac{1}{2} \frac{\bar{g}_{1}^{0}}{g_{1}^{0}} L^{3} \sin ^{6} \theta+\frac{3}{4}\left(\frac{\bar{g}_{1}^{0}}{g_{1}^{0}}\right)^{2} L^{6} \sin ^{12} \theta+\right. \\
& \left.2 \sqrt{3} \frac{\bar{g}_{2}^{1}}{g_{1}^{0}} L^{4}\left(\frac{\sin ^{4} \theta}{7}-\frac{\sin ^{9} \theta}{3}\right) \cos \phi_{0}\right\} \\
\phi= & \phi_{0}+\frac{\sqrt{3}}{7} \frac{\bar{g}_{2}^{1}}{g_{1}^{0}} L^{4} \sin ^{7} \theta \sin \phi_{0} .
\end{aligned}
$$

Equation (6a) gives the radial position of a field line point of colatitude $\theta$, and the longitude $\phi$ is counted from the noon meridian. In this equation one recognizes a dipole term which corresponds to the magnetic field of the Earth. The other terms between the brackets represent the perturbation components of the dipole field line, i.e. a uniform compression component, and an asymmetric compression component. Equation (6b) gives the deviation of the field line from a meridian plane. Figure 3 shows a real field line and its associated dipole field line. A bunch of particles would follow the real field line, and the directional fluxes would 


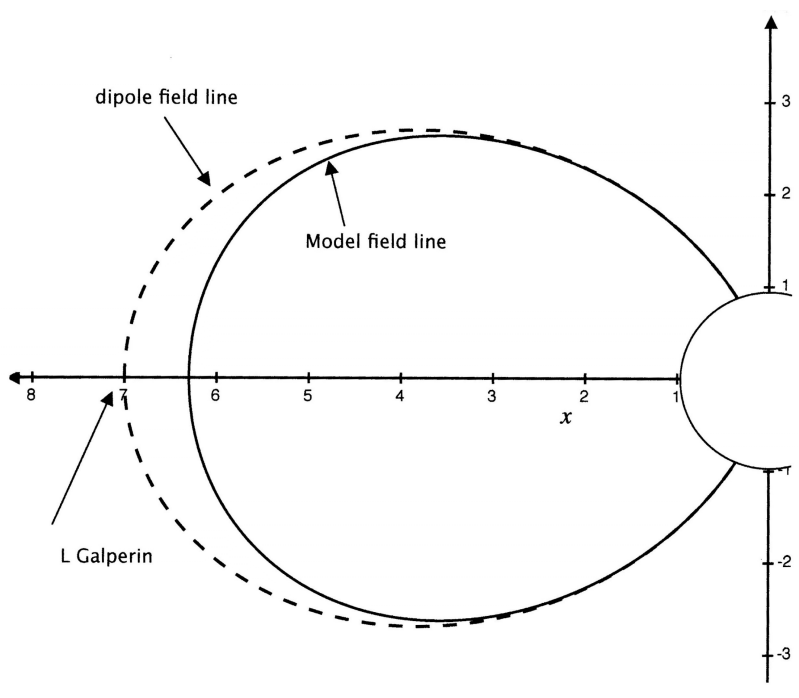

Fig. 3. The model field line is approximated by a dipole field line of label $L$, here the Galperin $L$ parameter. This dipole field line corresponds to the first term in Eq. (6a). The additional correcting terms in Eq. (6a) correspond to the distortion of the dipole magnetic field to give the model field line of label $L$.

be labelled with the parameter $L$ : the $L$ parameter of our real magnetic field line is clearly the Galperin $L$ parameter. There is a one-to-one correspondance between the Galperin $L$ parameter and the field line label.

The adiabatic invariants $I$ and $B_{m}$ can be expressed as a function of $L$, the mirror point colatitude $\theta_{m}$ and the longitude $\phi$. Taking into account the conservation of these invariants along a drift shell it is possible to express one variable as a function of the other two. This leads to the drift shell equation (Kosik, 1971a)

$L=L_{0}\left[1+\frac{\bar{g}_{2}^{1}}{g_{1}^{0}} L_{0}^{4} p\left(\theta_{m}\right)\left(\cos \phi-\cos \phi_{0}\right)\right]$,

where $L$ is the Galperin $L$ parameter at longitude $\phi$ and $L_{0}$ is the Galperin $L$ parameter at longitude $\phi_{0}$. In this equation $p\left(\theta_{m}\right)$ is a function of the mirror point colatitude $\theta_{m}$ of the particle.

If a convection electric field with potential $V=A L \sin \varphi$ is taken into account, where $A$ is assumed to be a constant equal to $2.5 \mathrm{kV} / \mathrm{L}$ in $L$ units, the drift shell equation is a little more complicated (Kosik, 1971b):

$L=L_{0}\left[1+\frac{\bar{g}_{2}^{1}}{g_{1}^{0}} L_{0}^{4} M\left(\theta_{m}\right)\left[\cos (\varphi-U)-\cos \left(\varphi_{0}-U\right)\right]\right.$,

where

$M=\sqrt{p^{2}\left(\theta_{m}\right)+\frac{A^{2} q^{2}}{E^{2}}\left|\frac{g_{1}^{0}}{\bar{g}_{2}^{1}}\right|^{2} \frac{g^{2}\left(\theta_{m}\right)}{L^{6}}}$ and $\tan U=\frac{A q}{E L^{3}} \frac{g_{1}^{0}}{\bar{g}_{2}^{1}} \frac{g\left(\theta_{m}\right)}{p\left(\theta_{m}\right)}$.

In these expressions $E$ is the kinetic energy of the particle and $p\left(\theta_{m}\right)$ and $g\left(\theta_{m}\right)$ are two functions which depend on the mirror point colatitude. Thus, $U$ depends on the relative strengths of the convection electric field and the asymmetric magnetic field component. Using again the conservation of the adiabatic invariants, the influence of a sudden compression of the magnetosphere on outer zone electron fluxes can also be studied (Kosik, 1973). All these results were obtained thirty years ago using the model of Mead and a linearization technique. It is therefore legitimate to question the domain of validity of these formulae. Near the Earth, for distances less than $4 \mathrm{Re}$, the contribution from the external magnetic field to the total field is negligible but this is not the case for the multipoles of the magnetic field of the Earth. Thus, the Galperin $L$ parameter will not differ from the McIlwain $L$ parameter. The formulae above will not apply as the internal magnetic field contributions from the mutipoles have been neglected. For distances greater than $7 \mathrm{Re}$, the Mead model, as well as the perturbation technique, do not give an adequate representation of the distant regions of the magnetosphere. Thus, the above formulae only apply to the synchronous region, typically for $L$ values between 5 and 7 .

\section{Discussion and conclusions}

McIlwain and Kerr (2002) have shown that the adoption of the Galperin $L$ parameter greatly facilitates the intercomparison of Cluster data with auroral displays. They have shown that the labelling of the field line is still possible when the observations are on open field lines. Darrouzet et al. (2004) have chosen the radial distance of the crossing point of the real field line with the geomagnetic equator as a label, in order to facilitate the analysis of the observations by the four Cluster spacecraft. In case of open field lines this type of labelling is not possible.

But, as we have seen, the Galperin $L$ parameter also has far reaching applications. Using the old and oversimplified Mead model we have shown that, in the synchronous region, the Galperin $L$ parameter is approximately the same as the $L$ parameter involved in the mathematical description of a model magnetosphere, i.e. in the field line equations and in the drift shells equations. Thus, besides its help in correlating distant observations, it offers the possibility of quantitatively describing the fluxes and the effects of the geomagnetic perturbations in the outer regions, taking into account convection electric fields, asymmetric magnetic fields and sudden compressions of the magnetosphere. Further mathematical work will be necessary to extend the mathematical description of the magnetosphere to more recent numerical models. More complex and more precise formulae than those presented here will be obtained. The domain of application of the new quantitative algorithms will extend from the range 
5-7 $\mathrm{Re}$ to the range 5-8 $\mathrm{Re}$, and enable a reasonnable quantitative guess of the processes further out in the magnetosphere. Thus, the usefulness of the Galperin $L$ parameter will be enhanced.

Acknowledgements. This paper resulted from the preparation of a new version of the Maglib software library for the CDPP. We greatfully acknowledge D. Boucon for her help in preparing the new version. We would like to thank C. Huc and C. Harvey for their encouragement and support in the updating of the software library. This revised version benefited from the comments of the two referees and precious advice from M. Schulz.

Topical Editor I. A. Daglis thanks S. W. H. Cowley and M. Hapgood for their help in evaluating this paper.

\section{References}

Darrouzet, F., Decreau, P. M., De Keyser, J., Masson, A., Gallagher, D. L., Santolik, O., Sandel, B. R., Trotignon, J. G., Rauch, J. L., Le Guirriec, E., Canu, P., Sedgemore, F., André, M., and Lemaire, J. F.: Density structures inside the plasmasphere: Cluster observations, Ann. Geophys., 22, 2577-2585, 2004, http://www.ann-geophys.net/22/2577/2004/.

Hakura, Y.: Tables and maps of geomagnetic coordinates corrected by the higher order spherical harmonic terms, Rep. Ionosph. Space Res. Japan, 19, 121-147, 1965.

Hapgood, M. A., Dimbylow, T. G., Sutcliffe, D. C., Chaizy, P. A., Ferron, P. S., Hill, P. M., and Tiratay, X. Y.: The Joint Science Operations Centre, Space Sci. Rev., 79, 487-525, 2004.

Hultquist, B.: The spherical harmonic development of the geomagnetic field, epoch 1945, transformed into rectangular geomagnetic coordinate systems, Ark. Geophys., 3, 53-61, 1958a.

Hultquist, B.: The geomagnetic field lines in higher approximation, Ark. Geophys., 3, 63-77, 1958b.
Kosik, J. C.: Mouvement des particules chargées dans un modèle analytique approché de la magnétosphère de Mead, Ann. Geophys., 27(1), 11-26, 1971a.

Kosik, J. C.: Motion of energetic particles in a magnetospheric model including a convection electric field, Planet. Space Sci., 19, 1209-1214, 1971b.

Kosik, J. C.: Influence of a sudden compression of the magnetosphere on outer zone electron fluxes measured at arbitrary pitchangle, Planet. Space Sci., 21, 1345-1353, 1973.

Kosik, J. C.: A quantitative model of the magnetosphere with poloidal vector fields, Ann. Geophys., 16, 1557-1566, 1998, http://www.ann-geophys.net/16/1557/1998/.

McIlwain, C. E.: Coordinates for mapping the distribution of magnetically trapped particles, J. Geophys. Res., 66(11), 3681-3691, 1961.

McIlwain, C. E. and Kerr, S. S.: Electric fields in the vicinity of $\mathrm{L}=20 \mathrm{Re}$ during substorms, in Sixth international conference on substorms, Un. Washington, Seattle, edited by: Winglee, R. M., 334-339, 2002.

Mead, G.: Deformation of the geomagnetic field by the solar wind, J. Geophys. Res., 69(7), 1181-1195, 1964.

Northrop, T. and Teller E.: Stability of the adiabatic motion of charged particle in the earth's field, Phys. Rev., 1, 215-225, 1960.

Roederer, J. G.: Dynamics of geomagnetically trapped radiation, Physics and Chemistry in Space, 2, Springer-Verlag, edited by: Roederer, J. G. and Zähringer, J., Denver, Heidelberg, 1970.

Prokhorenko, V.: Mission analysis for the interball project prelaunch orbit selection and long term experiment planning, in Interball mission and payload, RKA-IKI-CNES, 46-64, 1995.

Stasiewicz, K.: Polar cusp topology and position as a function of interplanetary magnetic field and magnetic activity: comparison of a model with Viking and other observations, J. Geophys. Res., 96, 15 789-15 800, 1991.

Tsyganenko, N. A.: A magnetospheric magnetic field model with a warped tail current sheet, Planet. Space Sci., 37, 5-20, 1989. 